\title{
Static Aeroelastic Analysis of Guided Slender Launch Vehicles
}

\author{
N.G. Humbad* \\ National Aeronautical Laboratory, Bangalore, India
}

\begin{abstract}
The static aeroelastic behavior of guided slender-body launch vehicles is explored by using a discrete system matrix method. An iterative procedure is used to calculate the effect of aerodynamic and eiastic force interactions. Neutral stability, divergence, and controltability of guided vehicles are discussed. The theory is applied to a research launch vehicle, and numerical results are given to show the effect of dynamic pressure and pitch rate on the pitch control force and center of pressure shift. The results indicate that excessive pitch control force can be avoided by selecting the flight dynamic pressure less than the dynamic pressure corresponding to neutral stability. Fin-control reversibility on account of the vehicle flexibility has been observed.
\end{abstract}

A

$A_{\text {e }}$

$(B M)$,

$C_{N_{\alpha}}$

$C_{N_{\alpha}} S$

$C_{N_{\alpha}} S_{\text {r }}$

D

E

$(E I)_{j}$

$F_{r}$

I

$L$

$M$

$m_{r}$

$N$

$P_{n}$

$P_{n_{e}}^{n}$

$q^{{ }^{n} r}$

$q_{\text {div }}$

$q_{F}$

$q_{n s}$

$r$

$r, s, i, j$

$u, v$

$W$

$x, y, z$

$x_{c g}$

\section{Nomenclature}

$=$ resultant aerodynamic force for elastic vehicle, $\mathrm{N}$

$=$ resultant of additional aerodynamic forces due to deformation of the vehicle, $\mathrm{N}$

$=$ bending moment at $j$ th station, $\mathrm{N}$-m

$=$ normal force coefficient slope

=product of total normal force coefficient slope and total reference area, $\mathrm{m}^{2} / \mathrm{rad}$

=product of normal force coefficient slope and panel area of the rth station, $\mathrm{m}^{2} / \mathrm{rad}$

$=$ reference body diameter of the vehicle, $\mathrm{m}$

=flexural modulus of elasticity, $\mathrm{N} / \mathrm{m}^{2}$

$=$ flexural rigidity at $j$ th station, $\mathrm{N}-\mathrm{m}^{2}$

$=$ resultant force at $r$ th station, $\mathrm{N}$

$=$ moment of inertia of cross-sectional area of structural members, $\mathrm{m}^{4}$

$=$ overall length of vehicle, $\mathrm{m}$

$=$ total mass of vehicle, $\mathrm{N}-\mathrm{s}^{2} / \mathrm{m}$

$=$ mass of $r$ th discrete element, $\mathrm{N}-\mathrm{s}^{2} / \mathrm{m}$

$=$ total number of discrete elements comprising analogous system

$=$ pitch control force at $n$th station, $\mathrm{N}$

$=$ additional pitch control force due to elasticity of the vehicle, $\mathrm{N}$

= pitch control force for rigid vehicle, $\mathrm{N}$

= dynamic pressure, $\mathrm{N} / \mathrm{m}^{2}$

= divergence dynamic pressure, $\mathrm{N} / \mathrm{m}^{2}$

$=$ flight dynamic pressure, $\mathrm{N} / \mathrm{m}^{2}$

=dynamic pressure corresponding to neutral stability for the elastic vehicle, $\mathrm{N} / \mathrm{m}^{2}$

= subscript defining discrete elements

$=$ integer variables

$=$ velocity components of origin of axes parallel to $x$ and $y$ axes, respectively, $\mathrm{m} / \mathrm{s}$

$=$ total centrifugal force, $\mathrm{N}$

$=$ coordinate system axes, $\mathrm{m}$

$=$ distance from $x$ origin to center of gravity, $\mathrm{m}$

Received Dec. 1976; revision received Oct. 14, 1977. Copyright (C) American Institute of Aeronautics and Astronautics, Inc., 1977. All rights reserved.

Index categories: Aeroelasticity and Hydroelasticity; LV/M Structural Design (including Loads).

* Scientist, Structural Sciences Division.

$$
\begin{aligned}
& x_{c p}, x_{c p}^{\prime} \\
& x_{e} \quad=\text { distance from } x \text { origin to center of } \\
& \begin{array}{l}
=\text { distance from } x \text { origin to center of } \\
\text { pressure of additional aerodynamic }
\end{array} \\
& \text { forces due to deformation of the } \\
& \text { vehicle, } \mathrm{m} \\
& x_{\text {ens }} \quad=x_{e} \text { at } q=q_{n s}, \mathrm{~m} \\
& Z_{j} \quad \text { section modulus, } \mathrm{m}^{3} \\
& \dot{\gamma} \quad=\text { pitching velocity of the vehicle about } z \\
& \text { axis, } \mathrm{rad} / \mathrm{s} \\
& \epsilon \quad=\text { accuracy } \\
& \alpha \quad=\text { angle of attack, rad } \\
& \alpha_{\text {div }} \quad=\alpha \text { at } q=q_{\text {div }} \text {, rad } \\
& \alpha_{e} \quad=\text { angle of attack due to elasticity of the } \\
& \text { vehicle, rad } \\
& \alpha_{n s} \quad=\alpha \text { at } q=q_{n s} \text {, rad } \\
& \alpha_{r} \quad=\text { angle of attack of rigid vehicle with } \\
& \text { airstream, rad } \\
& \theta_{r} \quad=\text { slope at } x=x_{r} \text { due to all of the loads }
\end{aligned}
$$

\section{Introduction}

TATIC aeroelasticity deals with the behavior of an elastic body or vehicle in an airstream wherein there is significant reciprocal interaction or feedback between the deformation and flow. Such interactions may tend to become smaller and smaller until a condition of stable equilibrium is reached, or they may tend to diverge and destroy the structure. Several different types of vehicles have demonstrated these aeroelastic problems by flight failures and anomalies. Of course, it is well known that launch vehicles are indeed flexible and the aeroelastic characteristics of launch vehicle structure tend to be very significant when the slenderness ratio $(L / D)$ is more than twenty. With the modern trend of designing highperformance, highly flexible flight vehicles, we are forced into areas where aeroelastic effects, which are ignored in rigidbody stability analysis, can and do cause structural and/or stability problems.

Guided launch vehicles are made to follow the predetermined flight path by using the pitch control force. In certain circumstances, the static aeroelastic interactions may influence the load distribution to such an extent that the maximum available pitch control force may become less than 
the required control force to guide the vehicle, thus making the vehicle unstable. Also, the structure may not withstand the load distribution calculated by accounting for aeroelastic interactions. Therefore, to ensure vehicle stability and structural integrity, it is essential to have a prior knowledge of static aeroelastic ef fects.

Several analytical methods are available for studying the aeroelastic characteristics of unguided launch vehicles. ${ }^{1-8}$ However, there seems a noticeable lag in the development of analytical methods for investigating the nonoscillatory stability problems of guided slender-body launch vehicles. This paper presents a systematic mathematical approach to the static aeroelastic analysis of such flight vehicles. The formulation is made by using a discrete system matrix method approach. The formulation and notations in this paper roughly follow that of Ref. 1. The presentation of the problem in matrix form has proved highly adaptable for highspeed digital computers. The method can be used in determining the static stability boundary for various combinations of system variables. Since the method is simple and direct, it may be of considerable help to the designer before he takes up the dynamic aeroelastic investigation.

A description of the method of calculating the pitch control force, load distribution, and center of pressure shifts on a slender, flexible vehicle in flight is presented. The phenomena of neutral stability, divergence, and controlability are discussed with the help of equilibrium equations. To illustrate the static aeroelastic behavior, an example application of the method to a research vehicle is submitted for various combination of system parameters. Several interesting features like neutral-stability control surface reversal are discussed with the help of a numerical example.

\section{Basic Assumptions}

The following assumptions are made in the static aeroelastic analysis of guided slender-body launch vehicles. It is assumed that elastic deformations of the vehicle body are described by elementary bending theory of beams. This procedure is justified for slender launch vehicles with $L / D \geq 20$. The effect of joint rotations is included in the analysis. All effects due to axial loads are considered to be negligible. The problem is restricted to the consideration of forces in the pitch plane of the vehicle. Centrifugal force due to continuous pitching is taken into account and is considered as the only inertia effect. The centripetal acceleration which gives rise to the centrifugal force is considered to act normal to the vehicle longitudinal body axis and to be constant over the vehicle span. This procedure is justified for small angles of attack, small elastic displacements, and when the length of the vehicle is small in comparison with the radius of curvature of the flight path. The acceleration due to gravity is excluded. It can, however, be incorporated very easily if the angle of the vehicle body axis to the vector of the Earth's gravitational acceleration is known, thus appropriately modifying the centripetal acceleration term.

The vehicle is assumed as nonspinning. The vehicle mass distribution is lumped at preassigned stations and lumped masses are assumed connected elastically by massless spring. Aerodynamic normal-force-coefficient slope distribution is also discretized.' The formulation treats the structure as being frozen in a nonoscillatory steady state of dynamic equilibrium, that is, the memory effect at a given instance of flight trajectory is not considered.

\section{Formulation of the Problem}

We consider a guided launch vehicle to be in a stable equilibrium under the action of the centrifugal force due to centripetal acceleration, aerodynamic forces, and a pitch control force. Figure 1 depicts a sketch of deflected slender launch vehicle. The coordinates $x$ and $y$ to the element are for body-fixed axes. The origin of $x$ and $y$ is taken near the nozzle

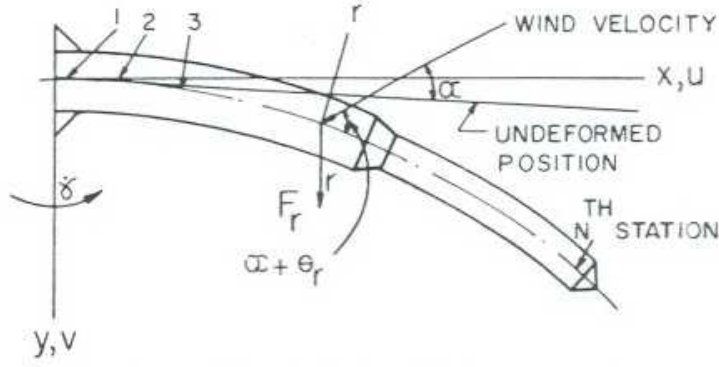

Fig. 1 Deformed slender launch vehicle.

end which is the first discrete element. Pitching velocity is chosen in the manner shown in Fig. 1. In view of the assumptions of planar motion and steady-state conditions the resultant force at the $r$ th element is the summation of transverse inertia force, $m_{r} u \dot{\gamma}$, and aerodynamic force, $q C_{N \alpha} S_{r}(\alpha+\theta)$, except at the station $n$ where the pitch control force is acting, in which case it is also included in the summation. Then

$$
F_{r}=m_{r} u \dot{\gamma}+q C_{N_{\alpha}} S_{r}\left(\alpha+\theta_{r}\right)+\delta_{r n} P_{n} \quad(r=1,2, \ldots, N)
$$

where

$$
\begin{array}{ll}
\delta_{r n} & =\text { Kronecker delta } \\
\delta_{r n} & =1 \text { for } r=n \\
\delta_{r n} & =0 \text { for } r \neq n
\end{array}
$$

where $n$ is the station at which pitch control force, $P_{n}$ is acting, $\alpha$ is the angle of attack of undeformed elastic vehicle, and $\theta_{r}$ is the slope at the rth station measured from the undeformed position.

Equation (1) can be conveniently written in matrix form for all values of $r$ from 1 to $N$ as

$$
\begin{aligned}
& \left.\left\{F_{r}\right\}=M u \dot{\gamma}\left\{\frac{m r}{M}\right\}+\alpha q \overline{C_{N_{\alpha}} S} \mid \frac{C_{N_{\alpha}} S_{r}}{\overline{C_{N_{\alpha}} S}}\right\rceil\{1\} \\
& \left.\quad+q \overline{C_{N_{\alpha}} S} \mid \frac{C_{N_{\alpha}} S_{r}}{\overline{C_{N_{\alpha}} S}}\right\rceil\left\{\theta_{r}\right\}+P_{n}\left\{\delta_{r n}\right\} \quad(r=1,2,3, \ldots, N)
\end{aligned}
$$

Let $\rho_{r, s}$ define the slope of the vehicle at $x=x_{r}$ due to a unit transverse load at $x=x_{5}$ when cantilevered at $x=0$. Then the slope at $x_{5}$ for a system of $N$ discrete masses can be written as

$$
\theta_{r}=\sum_{s=1}^{N} \rho_{r, s} F_{s}
$$

Equation (3) can be written in a matrix form as

$$
\left\{\theta_{r}\right\}=[\rho]\left\{F_{r}\right\}
$$

We obtain from Eqs. (2) and (4)

$$
\begin{aligned}
& \left.\left\{\theta_{r}\right\}=M u \dot{\gamma}[\rho]\left\{\frac{m_{r}}{M}\right\}+\alpha q \overline{C_{N_{\alpha}} S}[\rho] \mid \frac{C_{N_{\alpha}} S}{\overline{C_{N_{\alpha}} S}}\right]\{I\} \\
& \left.\quad+q \overline{C_{N_{\alpha}} S}[\rho] \mid \frac{C_{N_{\alpha}} S_{r}}{\overline{C_{N_{\alpha}} S}}\right\rceil\left\{\theta_{r}\right\}+P_{n}[\rho]\left\{\delta_{r n}\right\}
\end{aligned}
$$

Forces must satisfy the following two relationships for translational and rotational equilibrium:

$$
\begin{aligned}
& \lfloor l\rfloor\left\{F_{r}\right\}=0 \\
& \left\lfloor x_{r}\right\rfloor\left\{F_{r}\right\}=0
\end{aligned}
$$

It may be pointed out that the contribution of column forces in Eq. (7) is neglected in the foregoing discussion. This 
omission is acceptable by virtue of the assumption of small displacements as long as the thri'st remains well below the critical buckling thrust for column under free-free boundary conditions."

Substituting Eq. (2) in Eqs. (6) and (7), we get

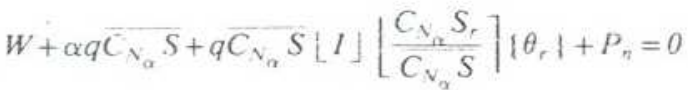

$$
\begin{aligned}
& W x_{c R}+\alpha q \bar{C}_{\mathrm{N}_{\alpha}} S x_{c p}+q \overline{C_{N_{\alpha}} S}\left\lfloor x_{r}\right\rfloor\left\lfloor\frac{C_{N_{\alpha}} S}{C_{N_{\alpha}} S}\right\rfloor\left\{\theta_{r}\right\rfloor+P_{n} x_{n}=0
\end{aligned}
$$

where

$$
\begin{aligned}
& \operatorname{Mu} \dot{\gamma}\lfloor l\rfloor\left\{\frac{m_{r}}{M}\right\}=W \\
& M u \dot{\gamma}\left\lfloor x_{r}\right\rfloor\left\{\frac{m_{r}}{M}\right\}=W x_{c g} \\
& {[1\rfloor\left\lfloor\frac{C_{N_{\alpha}} S_{r}}{\overline{C_{N_{\alpha}} S}}\right\rceil\{\}=1 \quad \text { since } \overline{C_{N_{\alpha}} S}=\sum_{r=1}^{N} C_{\mathrm{N}_{\alpha}} S_{r}} \\
& \left\lfloor x_{r}\right\rfloor\left\lfloor\frac{C_{N_{\alpha}} S_{r}}{C_{N_{\alpha}} S}\right\rceil\{\}=x_{c p}
\end{aligned}
$$

- The two unknowns-namely, the angle of attack of the undeformed vehicle to freestream $\alpha$, and the pitch control force $P_{n}$-are obtained from Eqs. (8) and (9) as follows:

$$
P_{n}=W\left(\frac{x_{c g}-x_{c p}}{x_{c p}-x_{n}}\right)+q C_{N_{\alpha}} S\left\lfloor\frac{x_{r}-x_{c p}}{x_{c p}-x_{n}}\right\rfloor\left\lfloor\frac{C_{N_{\alpha}} S_{r}}{C_{N_{\alpha}} S}\right\rceil\left\{\theta_{r}\right\}
$$

we define

$$
P_{n}=P_{n_{r}}+P_{n_{e}}
$$

where

$$
P_{n_{r}}=W\left(\frac{x_{c g}-x_{c p}}{x_{c p}-x_{n}}\right)
$$

and

$$
P_{n_{e}}=q \overline{C_{N_{\alpha}} S}\left\lfloor\frac{x_{r}-x_{c p}}{x_{c p}-x_{n}}\right\rfloor\left\lfloor\frac{C_{N_{\alpha}} S_{r}}{\overline{C_{N_{\alpha}} S}}\right\rceil\left\{\theta_{r}\right\}
$$

are the pitch control force for the rigid vehicle and the additional pitch control force for the elastic vehicle, respectively.

Similarly,

$$
\alpha=\frac{W}{q \overline{C_{N_{\alpha}} S}}\left(\frac{x_{n}-x_{c g}}{x_{c p}-x_{n}}\right)+\left\lfloor\frac{x_{n}-x_{r}}{x_{c p}-x_{n}}\right\rfloor\left\lfloor\frac{C_{N_{\alpha}} S_{r}}{\overline{C_{N_{\alpha}} S}}\right\rceil\left\{\theta_{r}\right\}
$$

we define

$$
\alpha=\alpha_{r}+\alpha_{e}
$$

where

$$
\alpha_{r}=\frac{W}{q \overline{C_{N_{\alpha}} S}}\left(\frac{x_{n}-x_{c q}}{x_{c p}-x_{n}}\right)
$$

and

$$
\alpha_{e}=\left\lfloor\frac{x_{n}-x_{r}}{x_{c p}-x_{n}}\right\rfloor\left\lfloor\frac{C_{N_{\alpha}} S_{r}}{C_{N_{\alpha}} S}\right\rceil\left\{\theta_{r}\right\}
$$

are the angle of attack of the rigid vehicle and the additional angle of attack of the elastic vehicle, respectively.

Substituting Eqs. (10) and (11) and in Eq. (5) and rearranging, we get

$$
\begin{aligned}
& \left\{\theta_{r}\right\}=\operatorname{Mu\dot {\gamma }}[\rho]\left\{\frac{m_{r}}{M}\right\}+\operatorname{Mu\dot {\gamma }}\left(\frac{x_{n}-x_{c g}}{x_{c p}-x_{n}}\right)[\rho]\left\lfloor\frac{C_{v_{\alpha}} S_{r}}{C_{v_{\alpha}} S}\right]\{H\} \\
& +\operatorname{Mu} \dot{\gamma}\left(\frac{x_{c p}-x_{c p}}{x_{c p}-x_{n}}\right)[\rho]\left\{\delta_{r n} i+q \bar{C}_{N_{\alpha}} S[\rho] \mid \frac{C_{N_{\alpha}} S_{r}}{\overline{C_{N_{\alpha}} S}}\right]\left\{\theta_{r}\right\} \\
& \left.+q \overline{C_{N_{\alpha}}} S[\rho]\left\{\hat{o}_{r n}\right\} \mid \frac{x_{r}-x_{c p}}{x_{\varepsilon p}-x_{n}}\right]\left\lfloor\frac{C_{N_{\alpha}} S_{r}}{C_{N_{\alpha}} S}\right\rceil\left\{\theta_{r}\right\} \\
& +q \overline{C_{N_{\alpha}} S}[\rho]\left\lfloor\frac{C_{\mathrm{V}_{\alpha}} S_{r}}{C_{\mathrm{N}_{\alpha}} S}\right\rceil \mid \|\left\lfloor\frac{x_{n}-x_{r}}{x_{c p}-x_{n}}\right\rfloor\left\lfloor\frac{C_{N_{\alpha}} S_{r}}{C_{N_{\alpha}} S}\right\rceil\left\{\theta_{r}\right\}
\end{aligned}
$$

Eq. (12) is the final equation to obtain slopes at various stations due to the aeroelastic interactions. The values of $\theta$, will converge asymptoticaily to stable equilibrium values if the dynamic pressure is less than the divergence dynamic pressure. Employing these values of $\theta_{r}$ 's in Eqs. (10) and (11) will yield the pitch control force and the angle of attack of the elastic vehicle in stable equilibrium, respectively.

It is obvious from the first three terms of Eq. (12) that the centrifugal force gives rise to a fixed angle of attack at various stations, whereas the last three terms of the equation show the interaction of the aerodynamic and the elastic forces. The problem of this interaction can be solved by assuming a set of $\left\{\theta_{r}\right\}$ initially. An iterative procedure is then carried out until convergence of $\theta$,'s is obtained with preset accuracy.

If $\theta_{r}{ }^{(i)}$ 's correspond to the values of $\theta_{r}$ 's after $i$ th iteration, then the iterations are stopped when $\theta_{r}{ }^{(i+l)}$ 's satisfy the following relation

$$
\left(\theta_{r}{ }^{(i+l)}-\theta_{r}^{(i)}\right) / \theta_{r}{ }^{(i)} \leq \epsilon \quad(r=2,3, \ldots, N)
$$

where $\epsilon$ is the preset accuracy, which in the present calculation is selected as $1 \%$. Since $\theta_{l}$ will always be zero because the structure is clamped at station one, value of index $r$ equal to one is not included.

From the rigid-body trajectory analysis of guided launch vehicles, the nominal value of pitch rate at any instance of flight is a known quantity. For static aeroelastic analysis, this pitch rate can be used in Eq. (12). Small perturbations from the nominal value of pitch rate do occur due to vehicle dynamics. However, if the maximum possible percentage variation of pitch rate from the nominal value is known approximately or assumed (say $\pm 10 \%$ ), then Eq. (12) can be solved by using the lower and the upper values of this pitch rate (i.e., $0.90 \dot{\gamma}$ and $1.10 \dot{\gamma}$ ) .

Numerical computations can be reduced considerably by taking the $x$ origin at the point of application of the pitch control force, since the third and the fifth term on the right side of Eq. (12) need not be calculated. It is also possible to solve Eqs. (5), (8), and (9) simultaneously for $\left\{\theta_{r}\right\}, P_{n}$, and $\alpha$ by machine inversion.

The resultant force at the $r$ th station, $F_{r}$, can be calculated from Eq. (1). It may then be employed to obtain the bending moment distribution along the vehicle. The bending moment at any station $j,(B M)_{j}$ may be calculated as

$$
(B M)_{j}=\sum_{r=1}^{j} F_{r} x_{r}
$$

Once the bending moment at each station is known, the bending stress at any station may be calculated by using the beam theory relationship

$$
\sigma_{j}=(B M)_{j} / Z_{j}
$$


where $Z_{j}$ is the section modulus at the $j$ th station.

Also, the location of the center of pressure for the elastic vehicle can be easily calculated from the following expression:

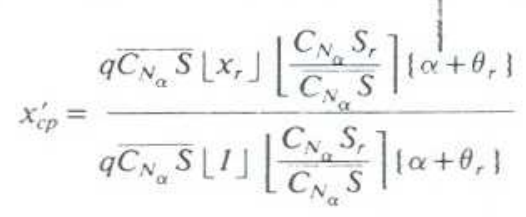

\section{Neutral Stability}

$x_{c p}^{\prime}$ moves forward with dynamic pressure due to aeroelastic interactions, thus reducing the static margin. The dynamic pressure at which the static margin vanishes (i.e. $x_{c p}^{\prime}$ coincides with $x_{c g}$ ) is called the dynamic pressure corresponding to neutral stability, $q_{n s}$. It is quite interesting that the pitch control force becomes zero at $q_{n s}$ irrespective of the magnitude of the centripetal acceleration of the vehicle. This fact can be ascertained from Eqs. (8) and (9). These equations can be rewritten as

$$
\begin{gathered}
W+A+P_{n}=0 \\
W x_{c g}+A x_{c p}^{\prime}+P_{n} x_{n}=0
\end{gathered}
$$

where

$$
A=q \overline{C_{N_{\alpha}} S}\lfloor l\rfloor\left\lfloor\frac{C_{N_{\alpha}} S_{r}}{\overline{C_{N_{\alpha}} S}}\right\rceil\left\{\alpha+\theta_{r}\right\}
$$

is the resultant aerodynamic force and $x_{c p}^{\prime}$ is its location from the $x$ origin.

Eliminating the unknown $A$ from Eqs. (16) and (17), and writing an equation for $P_{n}$, we get

$$
P_{n}=W\left(x_{c g}-x_{c p}^{\prime}\right) /\left(x_{c p}^{\prime}-x_{n}\right)
$$

Note that at $q=q_{n s}$ the static margin is zero, and therefore $P_{n}$ also becomes zero irrespective of the total centrifugal force. It is also seen that for a given value of flight dynamic pressure, the pitch control force is a linear function of centripetal acceleration. No pitch control force is required for zero centripetal acceleration. This is obvious, because the flight trajectory is then a rectilinear path (since $\dot{\gamma}=0$ ).

\section{Divergence}

The classical divergence occurs when changes in the angle of attack due to an incremental aerodynamic load give rise to bending deflections which in turn induce greater aerodynamic forces. These interactions diverge and destroy the structure. The dynamic pressure corresponding to this condition is called the divergence dynamic pressure. Mathematical insight into the phenomenon of divergence can be obtained by examining Eqs. (8) and (9). These equations can be rewritten as

$$
\begin{gathered}
W+\alpha q \overline{C_{N_{\alpha}} S}+A_{e}+P_{n}=0 \\
W x_{c g}+\alpha q \overline{C_{N_{\alpha}} S x_{c p}}+A_{e} x_{e}+P_{n} x_{n}=0
\end{gathered}
$$

where

$$
A_{e}=q \overline{C_{N_{\alpha}} S}\lfloor I\rfloor\left\lfloor\frac{C_{N_{\alpha}} S_{r}}{\overline{C_{N_{\alpha}} S}}\right\rceil\left\{\theta_{r}\right\}
$$

is the resultant of additional aerodynamic forces due to deformation of the vehicle and $x_{e}$ is its location from the $x$ origin. Eliminating $P_{n}$, we write

$$
A_{e}=\frac{W\left(x_{c g}-x_{n}\right)+\alpha q \overline{C_{N_{\alpha}} S}\left(x_{c p}-x_{n}\right)}{\left(x_{n}-x_{e}\right)}
$$

It is obvious from Eq. (23) that $A_{e}$ tends to infinity as $x_{e}$ tends to $x_{n}$. Thus, divergence occurs when the center of pressure of the additional aerodynamic forces coincides with the point of application of the pitch control force. On substitution of $x_{e}=x_{n}, q_{\mathrm{div}}$ can be obtained as

$$
q_{\mathrm{div}}=\frac{-W\left(c_{c g}-x_{n}\right)}{C_{N_{\alpha}} S \alpha_{\mathrm{div}}\left(x_{c p}-x_{n}\right)}
$$

$q_{n s}$ and $q_{\text {div }}$ are related by the following expression

$$
\frac{q_{\mathrm{div}}}{q_{n s}}=\frac{\left(x_{c g}-x_{n}\right)\left(x_{c p}-x_{e n s}\right)}{\left(x_{c g}-x_{e n s}\right)\left(x_{c p}-x_{n}\right)} \frac{\alpha_{n s}}{\alpha_{\mathrm{div}}}
$$

The above expression is implicit, since $x_{e n s}, \alpha_{n s}$, and $\alpha_{\mathrm{div}}$ are functions of dynamic pressure.

\section{Controllability}

It is seen from Eq. (19) that infinite control force is required when the center of pressure of the elastic vehicle coincides with the point of application of the pitch control force. Thus, the vehicle becomes unstable. However, in practice, the maximum available pitch control force determines the dynamic pressure range over which the vehicle is stable.

In the above discussion, the slope influence coefficient matrix may be obtained by using the procedure outlined by Ref. 9. However, it may be mentioned here that usually flexural rigidity for launch vehicles, is treated as being a linear function of $x$ between any two successive stations. Therefore exact integrals can be used in the calculation of slope influence coefficients rather than the numerical integration method suggested in Ref. 9.

Reference 10 has shown the numerical invariance of the transformation matrix of the equation of aeroelastic divergence of unguided launch vehicles. However, this is not applicable to Eq. (12) in the present case, because the eigenvector of $\theta_{r}$ 's obtained from Eq. (12) does depend on the location of the origin, whereas the eigenvector of $F_{r}$ 's of Ref. 10 does not depend on the choice of location of the origin.

\section{Results and Discussion}

Numerical calculations are made for a research launch vehicle. The input data such as flexural rigidity distribution, $E I(x)$, and joint rotation constants are taken from Tables I and II of Ref. 1; also the parameters $x_{r} / L, m_{r} / M$, and $C_{N_{\alpha}} S_{r} / C_{N_{\alpha}} S$ are taken from Fig. 6 of Ref. 1. The above data correspond to flight conditions at a Mach number of 4.0 and flight dynamic pressure $q_{F}$ of $323830 \mathrm{~N} / \mathrm{m}^{2}$. In the foregoing analysis, the vehicle is assumed to be guided along a given trajectory with a pitch rate $\dot{\gamma}$ such that centripetal acceleration $u \dot{\gamma}=-6.5 \mathrm{~m} / \mathrm{s}^{2}$. The pitch control force is assumed to act at station number one which is the zeroth station of Ref. 1 . The calculations are made for various percentages of flight dynamic pressure and centripetal acceleration. In the present calculation $\epsilon$ is selected as $1 \%$.

Figure 2 depicts the pitch control force variation as a function of percentage of flight dynamic pressure and for different values of centripetal acceleration. It is seen from the curves that the absolute value of the pitch control force decreases with an increase in the dynamic pressure, until it reaches zero. Afterwards, there is a sharp increase in the pitch control force requirements. It is clear from the curves that any increase in the centripetal acceleration requires an increase in the control force. It is quite interesting that the change in the control force per unit change in $u \dot{\gamma}$ at a given $q$ decreases as the dynamic pressure increases and becomes zero at $q=1.6$ $q_{F}$. This is because the elasticity of the vehicle alters the aerodynamic load distribution, and the center of pressure (cp) moves forward. For a rigid vehicle, $c p$ is behind the center of gravity $(c g)$ and, therefore, as the distance between $c p$ and $c g$ decreases, the smaller pitching moment at $c g$ can be balanced 


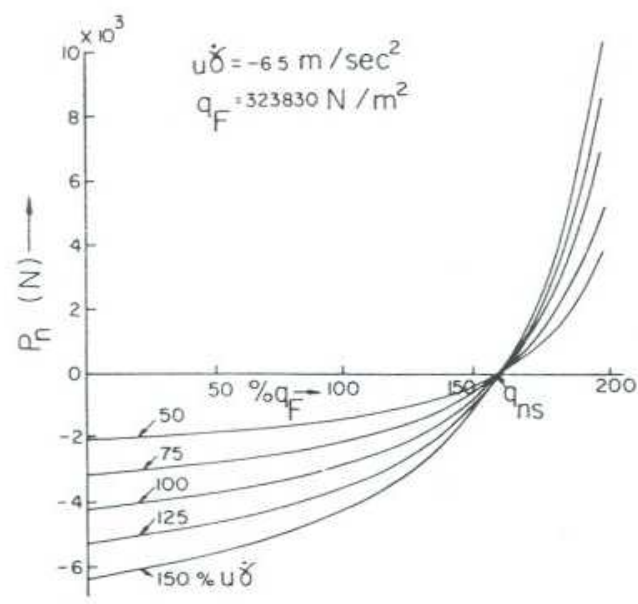

Fig. 2 Pitch control force variation with dynamic pressure and centripetal acceleration.

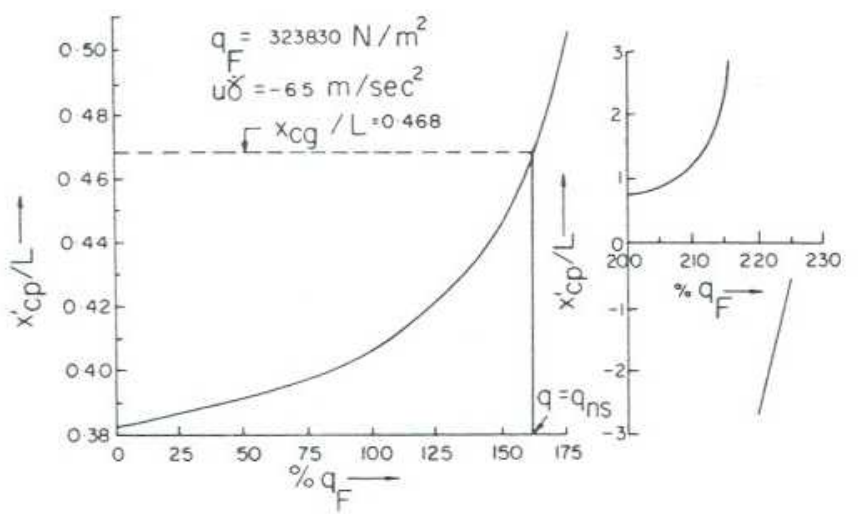

Fig. 3 Center-of-pressure shift with dynamic pressure.

by a smaller pitch control force at the nozzle end. When $c p$ coincides with $\mathrm{cg}$ then, of course, the vehicle does not need any control force since there is no pitching moment at $\mathrm{cg}$. It may be noted further that, at this stage, inertia forces exactly balance the aerodynamic forces to satisfy force and moment equilibrium conditions for the free-free structure. And this is the explanation of why all the curves with different $u \dot{\gamma}$ pass through a single point at $q=1.6 q_{F}\left(=q_{n s}\right.$ where $q_{n s}$ is the dynamic pressure corresponding to the neutral stability of the vehicle). This confirms the conclusion drawn earlier from Eq. (19). For $q>q_{n s}$, however, the centrifugal force has a destabilizing effect on the pitching moment, and therefore the pitch control force curves become steeper with smaller increases in dynamic pressure than when $q<q_{n s}$. The working range of dynamic pressure may be selected below the dynamic pressure corresponding to the neutral stability of the vehicle. Allowing about $20 \%$ margin of safety, the flight dynamic pressure $q_{F}$ may be selected as $q_{F} \leq 0.80 q_{n s}$.

The center of pressure of elastic vehicle is calculated by using Eq. (15) for various values of flight dynamic pressures and is plotted in Fig. 3. The fact that $c p$ coincides with $c g$ at $q=1.6 q_{F}$ is confirmed from the plot. Beyond twice the flight dynamic pressure, several interesting features regarding the location of the center of pressure are observed. A little beyond twice $q_{F}$, the $c p$ lies ahead of the vehicle. This is quite possible because we are considering the point of application of the resultant of aerodynamic loads only and not the overall equilibrium of all the forces, viz. control, aerodynamic, and centrifugal forces. Another interesting feature is that between $2.15 q_{F}$ and $2.20 q_{F}, c p$ moves from upstream of the vehicle and goes far downstream of the vehicle. To illustrate this feature $x_{c p}^{\prime} / L$ is plotted separately for the dynamic pressure range of $2.0 q_{X}$ to $2.25 q_{F}$. The explanation of this behavior is
Table 1 Resultant aerodynamic forces at various stations at dynamic pressures of $2.15 q_{F}$ and $2.20 q_{F}$

\begin{tabular}{|c|c|c|c|c|}
\hline \multirow{2}{*}{$\begin{array}{l}\text { Station } \\
\text { number } \\
(r)\end{array}$} & \multirow{2}{*}{$\frac{x_{r}}{L}$} & \multirow{2}{*}{$\frac{C_{N_{\alpha}} S_{r}}{\overline{C_{N_{\alpha}} S}}$} & \multicolumn{2}{|c|}{$\begin{array}{c}\text { Resultanty } \\
\text { aerodynamic force at }\end{array}$} \\
\hline & & & $2.15 q_{F}{ }^{3}, N$ & $2.20 q_{F}, \mathrm{~N}$ \\
\hline 1 & 0.0 & .3385 & -6126 & -10745 \\
\hline 2 & 0.0706 & .0096 & -0198 & -0338 \\
\hline 3 & 0.1312 & .0059 & -0126 & -0213 \\
\hline 4 & 0.1918 & .0084 & -0183 & -0309 \\
\hline 5 & 0.2473 & .0063 & -0139 & -0235 \\
\hline 6 & 0.2977 & .0092 & -0203 & -0344 \\
\hline 7 & 0.3482 & .0093 & -0206 & -0347 \\
\hline 8 & 0.4037 & .0174 & -0372 & -0632 \\
\hline 9 & 0.4570 & .2612 & 0954 & -0536 \\
\hline 10 & 0.4945 & .0144 & 0003 & -0097 \\
\hline 11 & 0.5288 & .0123 & -0178 & -0337 \\
\hline 12 & 0.5652 & .0229 & -0371 & -0687 \\
\hline 13 & 0.6056 & .0234 & -0324 & -0632 \\
\hline 14 & 0.6459 & .0357 & -0150 & -0500 \\
\hline 15 & 0.6762 & .0130 & -0030 & -0151 \\
\hline 16 & 0.7004 & .0511 & 0692 & 0490 \\
\hline 17 & 0.7267 & .0204 & 0287 & 0203 \\
\hline 18 & 0.7509 & .0072 & 0016 & -0049 \\
\hline 19 & 0.7822 & .0085 & -0025 & -0124 \\
\hline 20 & 0.8124 & .0158 & 0087 & -0050 \\
\hline 21 & 0.8478 & .0234 & 0616 & 0588 \\
\hline 22 & 0.8902 & .0610 & 6234 & 7873 \\
\hline 23 & 0.9356 & .0250 & 2957 & 3767 \\
\hline
\end{tabular}

${ }^{\mathrm{a}} u \dot{\gamma}=-6.5 \mathrm{~m} / \mathrm{s}^{2}, q_{F}=323,830 \mathrm{~N} / \mathrm{m}^{2}$

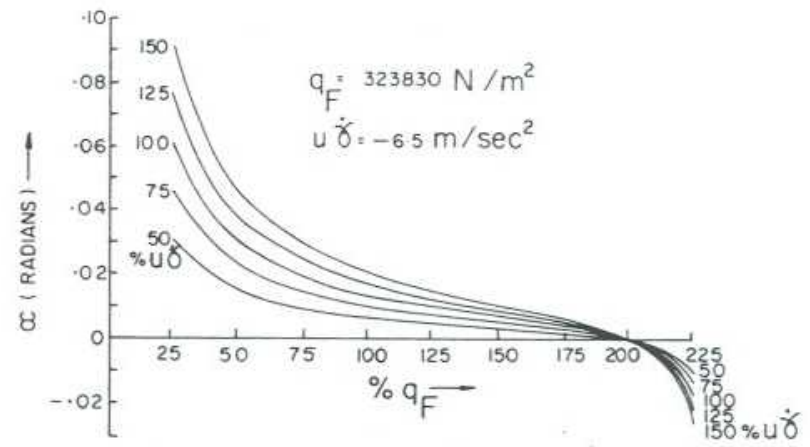

Fig. 4 Angle-of-attack variation with dynamic pressure and centripetal acceleration.

easily found in Table 1 , in which the aerodynamic forces are tabulated at $q=2.15 q_{F}$ and $2.20 q_{F}$. It is seen from the table that station 9 is a second dominant lift panel (second-stage fins), and therefore the sign of the angle of attack of the panel to airstream has major effect in determining the $c p$ location. At $q=2.15 q_{F}$ the angle of attack at station 9 is still positive, therefore $c p$ remains ahead of the vehicle. But as soon as the angle of attack at the ninth station becomes negative at $q=2.20 q_{F}, x_{c p}^{\prime}$ becomes negative.

Figure 4 depicts the angle of attack at the first station of the undeformed elastic vehicle as a function of dynamic pressure and for different values of the centripetal accelerations. It is quite interesting that all the curves with different $u \dot{\gamma}$ pass through the $x$ axis at a dynamic pressure of about $2.0 q_{F}$. This has significant importance as the angle of attack at the first station becomes negative for values of dynamic pressures greater than twice the flight dynamic pressure, and therefore the dominant lift panel (i.e., the first-stage fins at the first station) has a destabilizing effect. The classical terminology of control surface reversal can now be applied with the exception that the control surface reversal occurs here due to the vehicle body flexibility rather than the flexibility of control surface.

Figure 5 depicts the bending moment variation along the span of the vehicle at $u \dot{\gamma}=-6.5 \mathrm{~m} / \mathrm{s}^{2}$ and for different values 


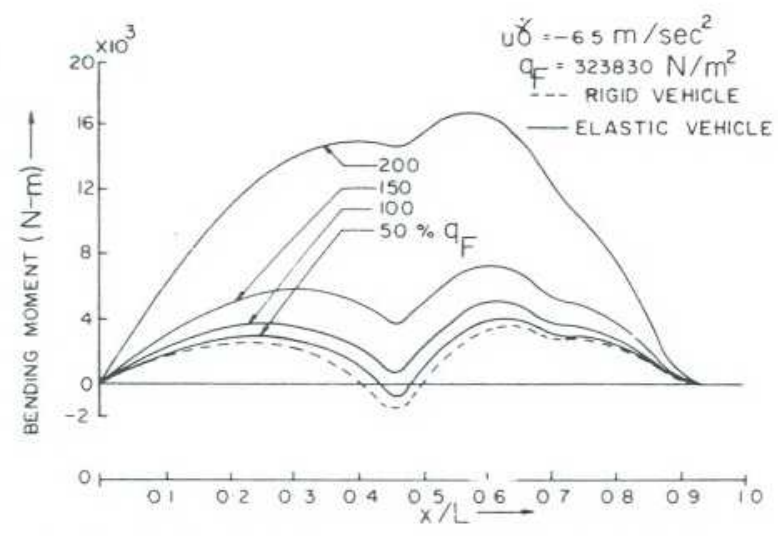

Fig. 5 Lengthwise bending moment variation with dynamic pressure.
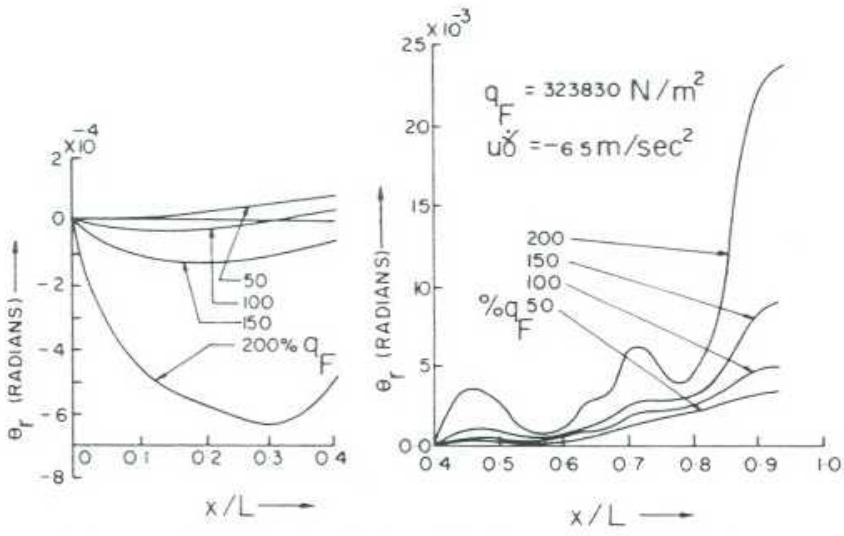

Fig. 6 Lengthwise slope variation with dynamic pressure.

of the flight dynamic pressure. The dotted curve corresponds to the bending moment variation along the span of the rigid vehicle at $u \dot{\gamma}=-6.5 \mathrm{~m} / \mathrm{s}^{2}$, which does not depend on the value of dynamic pressure. It is clearly seen that the flexibility has a significant effect on the bending moment/load distribution. It may be noted that the bending moment increases sharply after about $1.60 q_{F}$, and that the point at which the maximum bending moment occurs moves forward, towards the nosecone end of the vehicle, with an increase in the dynamic pressure.

Figure 6 depicts the slope distribution along the length of the vehicle for different values of the flight dynamic pressure. At very low dynamic pressures the slope distribution resembles the first mode shape of vibration of a cantilever beam. As the dynamic pressure increases, the slopes remain negative in the region of $x / L=0$ to 0.40 , indicating that the vehicle deformation resembles the second mode shape of vibration of a cantilever beam.

\section{Conclusions}

The equations essential for performing aeroelastic analysis of guided slender launch vehicles have been presented. Neutral stability, divergence, and controllability of guided vehicles are discussed with the help of equilibrium equations. Static aeroelastic effects such as pitch control force, bending moment variation, etc. with dynamic pressure and centripetal acceleration are presented and discussed for a research launch vehicle. Present analysis shows that it is desirable to keep the flight dynamic pressure less than the dynamic pressure corresponding to neutral stability in order to avoid excessive pitch control force. Fin control reversibility due to vehicle flexibility has been observed during the investigation.

\section{Acknowledgments}

The author is grateful to B.R. Somashekar, Head of the Structural Sciences Division, and S. Durvasula of the Indian Institute of Science, Bangalore, for helpful discussions. The author expresses his sincere thanks to A.K. Godbole who widened the viewpoint on many points of fact during the computational aspects.

\section{References}

'Alley, V.L. Jr. and Gerringer, A.H., "An Analysis of Aeroelastic Divergence in Unguided Launch Vehicles," TN-D-3281, NASA, March 1966

${ }^{2}$ Thomson, K.D. " Aeroelastic Divergence of Slender Multistage Test Vehicles," Weapons Research Establishment, South Australia, TN HSA-93, Nov, 1962

${ }^{3}$ O'Keefe, D.A., "General Static Aeroelastic Analysis for a Body of Revolution," AlAA Journal, Vol. 3, Dec, 1965, pp. 2257-61.

${ }^{4}$ Young, C.P. Jr., "A Numerical Method for Determining the Aeroelastic Divergence Characteristics of Unguided, Slender-Body, Multistage Launch Vehicles," NASA TN-D-3893, April 1967.

${ }^{5}$ Baines, D.J. and Pearson, K.G., "Aeroelasticity as a Consideration in Aerodynamic Design of Rolling, Unguided Research Vehicles," Journal of Spacecraft and Rockets, Vol. 4, Dec. 1967, pp. 1603-1608.

${ }^{6}$ Young, C.P. Jr., "Analysis of the Aeroelastic Divergence of Two Experimental Unguided Launch Vehicles," NASA TN-D-4846, Nov 1968.

${ }^{7}$ Young, C.P. Jr., "On the Steady Aeroelastic Behaviour of a Spinning Rocket Vehicle Having Aerodynamic Asymmetry,' presented at the AIAA 2nd Sounding Rocket Vehicle Technology Conference, Williamsburg, Va., Dec. 7-9, 1970.

${ }^{8}$ Mayers, S.C., "Aeroelastic Analysis of Sounding Rocket Vehicles," AIAA Paper 73-284, Albuquerque, N.M., March 1973.

${ }^{9}$ Alley, V.L. Jr. and Gerringer, A.H., "A Matrix Method for the Determination of the Natural Vibrations of Free-Free Unsymmetrical Beam with Application to Launch Vehicles," NASA TN-D-1247, 1962.

${ }^{10}$ Humbad, N.G., "Comment on the Equation for Aeroelastic Divergence of Unguided Launch Vehicles," AIAA Journal, Vol. 12, Dec. 1974, pp. 1739-1740. 